\title{
Critical Role of the Capsaicin-Sensitive Nerve Fibers in the Development of the Causalgic Symptoms Produced by Transecting Some but Not All of the Nerves Innervating the Rat Tail
}

\author{
Yang In Kim, Heung Sik Na, Jung Soo Han, and Seung Kil Hong \\ Neuroscience Research Institute and Department of Physiology, College of Medicine, Korea University, Seoul, \\ Korea 136-705
}

\begin{abstract}
We investigated the role of capsaicin-sensitive small diameter fibers in the development of the thermal and mechanical allodynia in a new rat model for neuropathic pain, produced by transecting some but not all of the nerves innervating the tail. Capsaicin $(50 \mathrm{mg} / \mathrm{kg}, \mathbf{s . c}$.) injected neonatally prior to the nerve injury produced thermal hypoalgesia in the tail the degree of which was variable across individual rats, presumably as a result of variable degeneration of the small diameter fibers. When subjected to the nerve injury, the animals with moderate thermal hypoalgesia exhibited signs of pain (e.g., tail flick) to normally innocuous mechanical stimuli applied to the tail with von Frey hairs (4.9 mN or $19.6 \mathrm{mN}$ bending force), but not to thermal stimuli given by immersion of the tail into cold $\left(4^{\circ} \mathrm{C}\right)$ or warm $\left(40^{\circ} \mathrm{C}\right)$ water. The animals with marked thermal hypoalgesia, on the other hand, exhibited no signs of pain either to the mechanical or to the thermal stimuli. These results suggest that the capsaicin-sensitive fibers are critical in the development of both the mechanical and thermal allodynia. It is hypothesized that the destruction of Aס- and C-nociceptive fibers by capsaicin prevented activities induced in these fibers by the nerve injury from producing a central sensitization and thus allodynia.
\end{abstract}

[Key words: capsaicin, allodynia, causalgia, neuropathic pain, spinal cord, C-fibers, animal model]

Peripheral nerve injury sometimes results in chronic neuropathic pain such as causalgia (Mitchell, 1872; Richards, 1967; Tahmoush, 1981; Blumberg and Janig, 1983; Devor, 1983). This type of pain is characterized by spontaneous burning pain accompanied by hyperalgesia and allodynia lasting variable times. At present, the mechanisms underlying the causalgic symptoms are not completely understood.

Recently, three different experimental animal models for causalgia, produced by a partial injury of the nerves supplying the rat hind paw, were developed by Bennett and Xie (1988), Seltzer et al. (1987, 1990) and Kim and Chung (1991, 1992), respectively. Although these models are attractive in that the characteristic symptoms of causalgia such as allodynia and hyperal-

\footnotetext{
Received July 15, 1994; revised Jan. 9, 1995; accepted Jan. 17, 1995.

We thank Young Hoon Kim for photographic help. This article was sup ported by Non Directed Research Fund, Korea Research Foundation.

Correspondence should be addressed to Heung Sik Na, M.D., Ph.D., De partment of Physiology, College of Medicine, Korea University, Seoul, Korea 136-705.

Copyright 1995 Society for Neuroscience $0270-6474 / 95 / 154133-07 \$ 05.00 / 0$
}

gesia are clearly observed, they appear to suffer from some drawbacks; behavioral tests using the hind paw are difficult to perform and the deformity of the hind paw following the nerve injury does not allow blind behavioral tests. To avoid these potential problems we have developed a new rat model $(\mathrm{Na}$ et al., 1994), produced by transecting some but not all of the nerves innervating the rat tail. This model, similar to the previously developed ones, displays chronic neuropathic symptoms like mechanical and thermal (cold and warm) allodynia. Furthermore, the model offers two major advantages in performing the behavioral tests. First, application of both mechanical and thermal stimuli to the partially denervated area (i.e., the tail) is easy. Second, blind behavioral tests are possible due to the lack of deformity in the tail after the nerve injury.

In the present study, using our new model, we assessed whether or not small diameter afferents (i.e., $\mathrm{A} \delta$ - and $\mathrm{C}$-fibers) are critical in the development of causalgic symptoms. The role of these fibers was inferred by examination of the presence of causalgic symptoms after depleting the fibers with neonatal subcutaneous injection of capsaicin, a treatment known to produce irreversible degeneration of most (up to $80 \%$ ) $\mathrm{C}$-fibers and some A $\delta$-fibers (Lawson and Nickels, 1980; Nagy et al., 1981; Nagy and Van der Kooy, 1983). Inconsistent with the previous report that neonatal capsaicin treatment prevented the development of thermal hyperalgesia, but not mechanical allodynia and hyperesthesia (Shir and Seltzer, 1990), our results indicate that mechanical, as well as thermal, allodynia is dependent on the capsaicin-sensitive fibers.

\section{Materials and Methods \\ Capsaicin treatment}

Twenty-two male Sprague-Dawley rats were injected subcutaneously within $48 \mathrm{hr}$ of birth with capsaicin $(50 \mathrm{mg} / \mathrm{kg}$, Sigma, dissolved in saline containing $10 \%$ Tween 80 and $10 \%$ ethyl alcohol). Twelve other rats were injected with the vehicle only. After being weaned, the rats were reared in individual or group cages. When young adults, weighing $100-200 \mathrm{gm}$ (7-8 weeks old), the animals were subjected to further experimental manipulations (below).

\section{Assessment of capsaicin effects}

Capsaicin treatment has been reported to produce permanent thermal hypoalgesia (Holtzer et al., 1979; Jansco and Jansco-Gabor, 1980; Nagy et al., 1980; Gamse, 1982; Nagy and Van der Kooy, 1983; Doucette et al., 1987; but see Hayes et al., 1981a,b; Cervero and McRitchie, 1981, 1983; Buck et al., 1982; Hara et al., 1981; Meller et al., 1992). This abnormality is presumed to be due to destruction by capsaicin of Cand/or A $\delta$-afferents in the peripheral nerves (Lawson and Nickels, 1980).

To indirectly assess the efficacy of the neonatal capsaicin treatment 


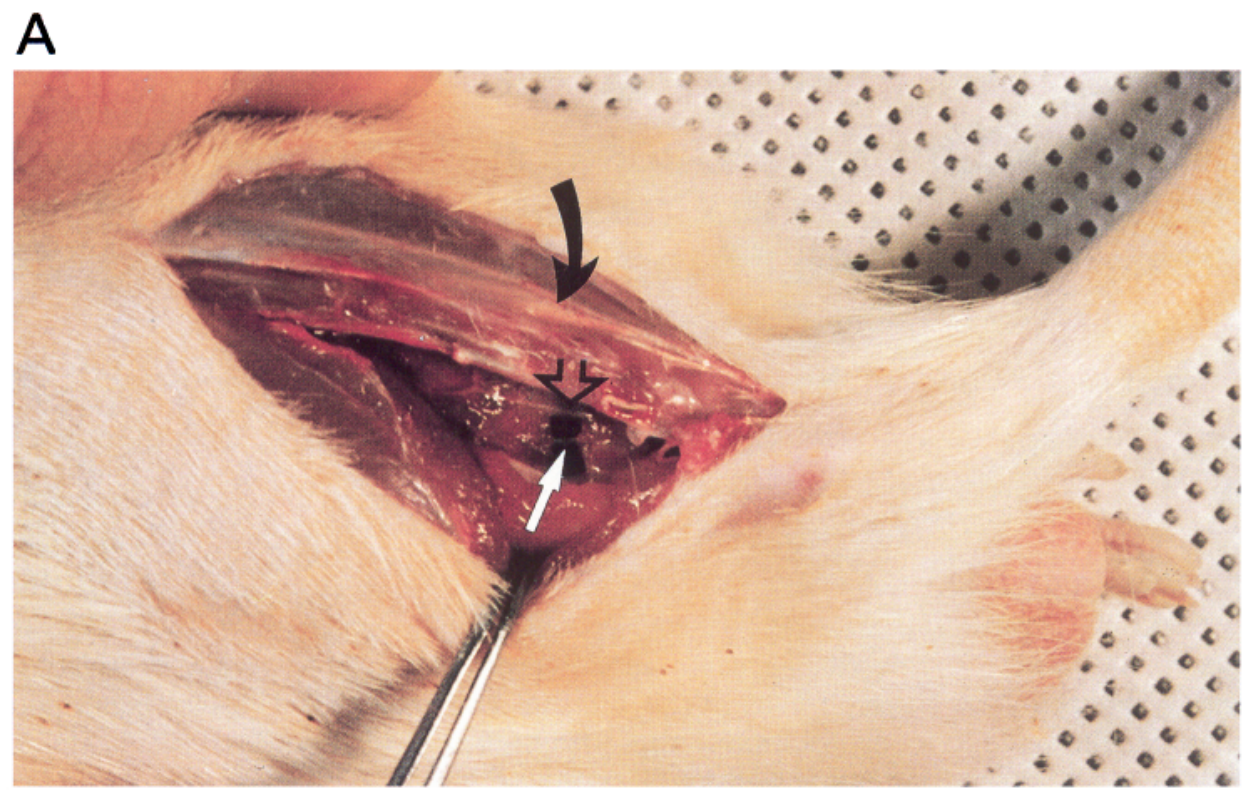

B

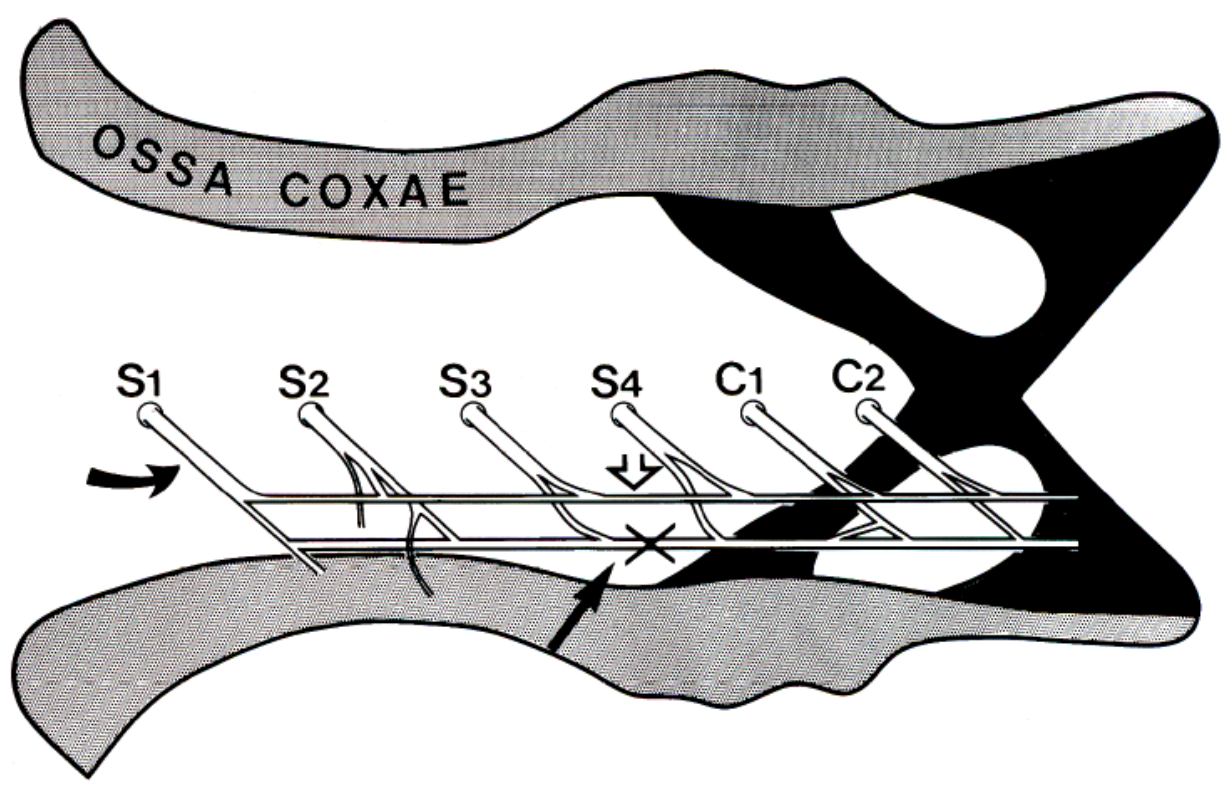

Figure 1. A, A photograph showing the surgically exposed inferior (white arrow) and "superior" (open arrow) caudal trunks. A small piece of black vinyl was placed under the trunks for better viewing of these structures. The curved arrow indicates the spinous process of the S4 vertebra. $B$, A schematic diagram (dorsal view) illustrating how the inferior (black arrow) and "superior" (open arrow) caudal trunks are composed of and the level of the transection $(X)$ of the inferior caudal trunk. The curved arrow indicates the $\mathrm{S} 1 \mathrm{spi}-$ nal nerve. The vertebrae and spinal cord are not shown in this diagram. in destroying $\mathrm{C}$ - and $\mathrm{A} \delta$-fibers, we compared the responses of the capsaicin- and vehicle-injected animals to thermal stimulation given by immersing the tail into $48^{\circ} \mathrm{C}$ water. The latency of the tail-withdrawal response to the thermal stimulation was measured with a cut-off time of $30 \mathrm{sec}$. This was repeated five times at a $5 \mathrm{~min}$ interval to give rise to an average value for each animal.

\section{“Neuropathic"' surgery}

The capsaicin- or vehicle-treated rats were subjected to the "neuropathic" surgery, as previously described by Na and his coworkers (1994). In short, under sodium pentobarbital anesthesia $(40 \mathrm{mg} / \mathrm{kg}$, i.p.), the left inferior caudal trunk was exposed freed carefully from the surrounding tissues and transected at the level between the S3 and S4 spinal nerves. To prevent the possible rejoining of the proximal and distal ends of the severed trunk, about $2 \mathrm{~mm}$ piece of the trunk was removed from the proximal end. This surgery eliminated the S1-S3 spinal nerve innervation of the tail via the left inferior caudal trunk. Figure $1 A$ shows the surgically exposed inferior and "superior" caudal trunks, and Figure $1 B$ illustrates schematically how these trunks are composed of and the level of the transection of the inferior caudal trunk.

\section{Behavioral tests for signs of neuropathic pain}

The presence of the behavioral signs suggestive of neuropathic pain (i.e., mechanical, cold and warm allodynia) was examined $1 \mathrm{~d}$ prior to and $1,4,7$, and $14 \mathrm{~d}$ after the "neuropathic" surgery. All of these behavioral tests were conduced by one investigator who was uninformed of whether or not the animal was neonatally treated with capsaicin; nevertheless, the investigator could tell whether or not the animal was treated with capsaicin by the presence or absence of the wounds and scars on the animal's face and body (see Results).

Mechanical allodynia. The signs suggestive of mechanical allodynia were sought by applying normally innocuous mechanical stimuli to the tail using von Frey hair with the bending force of $4.9 \mathrm{mN}(0.5 \mathrm{gm})$ or $19.6 \mathrm{mN}(2.0 \mathrm{gm})$ (made of horse tail hairs, shank diameters of about 150 and $200 \mu \mathrm{m}$; cylindrical tip shape). For convenient application of the stimuli the rat was restrained in a transparent plastic tube and the tail was laid on a wooden plate. The most mechanically sensitive spot of the tail was first determined using the $4.9 \mathrm{mN}$ von Frey hair; initially the most sensitive area was determined by rubbing various areas of the tail with the shank of the von Frey hair, and then, this area was poked systematically with the von Frey hair to locate the most sensitive spot. 
This spot was marked with a sharp marking pen. The actual test was conducted by poking gently (not jabbing) the spot with the von Frey hair. An abrupt tail movement of about $0.5-20 \mathrm{~cm}$ in response to the von Frey hair stimulation was considered to be an abnormal response suggestive of mechanical allodynia. Whereas, lack of or a slow, minute $(<0.1 \mathrm{~cm})$ tail movement was considered to be a normal response, indicating the absence of mechanical allodynia. The determination of the abnormal and normal tail responses was made subjectively by the investigator. In the present study we did not attempt to rate the abnormal tail responses, because (1) these responses having a large variability in magnitude could not be subdivided with certainty into a few classes and (2) the average magnitude of the responses appeared not different across individual animals within a given experimental group and between vehicle- and capsaicin-treated animal groups.

The von Frey test was repeated 10 times at a $10-20 \mathrm{sec}$ interval for each animal on each experimental day. During the repeated trials, the test stimuli were delivered to the same spot with no difficulties, since the tail was usually stationary. The repetition of the test was to determine the frequency of the abnormal tail responses (expressed in percentage of trials), an index adopted to follow the course of the development of mechanical allodynia following the "neuropathic" surgery.

After the first series of tests with the $4.9 \mathrm{mN}$ von Frey hair, tests with the $19.6 \mathrm{mN}$ hair ensued; the most sensitive spot determined with the $4.9 \mathrm{mN}$ hair was again stimulated.

Thermal allodynia. The signs suggestive of thermal allodynia were sought by immersing the tail in $4^{\circ} \mathrm{C}$ or $40^{\circ} \mathrm{C}$ water. The rat was restrained in a plastic tube, and the tail was drooped for convenient application of the thermal stimuli. Following the tail immersion, the investigator continuously observed the tail to see if it moved abruptly and measured the latency of the tail movement with a cut-off time of 30 sec. An abrupt tail movement of about $0.5-20 \mathrm{~cm}$ (ranging from small tail twitch in the water to tail withdrawal out of the water) with a latency shorter than the cut-off time was considered to be an abnormal tail response suggestive of thermal allodynia. Whereas, lack of tail movement until the cut-off time or a slow, minute $(<0.2 \mathrm{~cm})$ tail movement within the cut-off time was considered to be a normal response, indicating the absence of thermal allodynia. The determination of the abnormal and normal tail responses was mate subjectively by the investigator. In this study we did not attempt to rate the abnormal tail re sponses, because (1) these responses having a large variability in magnitude could not be subdivided with certainty into a few classes and (2) the average magnitude of the responses appeared not different across individual animals within a given experimental group and between vehicle- and capsaicin-treated animal groups.

The tail immersion test was repeated five times at a $5 \mathrm{~min}$ interval to obtain the average tail response latency for each animal on each experimental day. In calculating the average latency, the cut-off time (i.e., $30 \mathrm{sec}$ ) was assigned to the normal responses. The average latency was adopted as an index for the course of the development of thermal allodynia following the "neuropathic" surgery.

Tests with the $4^{\circ} \mathrm{C}$ water preceded the ones with the $40^{\circ} \mathrm{C}$ water by about an hour. The possibility that the abnormal tail responses to the $4^{\circ} \mathrm{C}$ or $40^{\circ} \mathrm{C}$ water were due to the mechanical contact of the tail with the water instead of thermal stimulation was essentially ruled out, since (1) in a vast majority of the cases (11 out of 12 animals), $30^{\circ} \mathrm{C}$ water did not elicit any abnormal tail responses and (2) the $4^{\circ} \mathrm{C}$ or $40^{\circ} \mathrm{C}$ waterelicited responses had latencies longer than at least a few seconds, unlike the von Frey hair-evoked responses which had virtually no latencies.

\section{Statistical analyses}

Friedman's rank test was used to determine whether or not an overall difference was present among the behavioral test scores of a given animal group obtained $1 \mathrm{~d}$ before and $1,4,7$, and $14 \mathrm{~d}$ after the "neuropathic" surgery. When this test indicated a significant difference, post hoc comparisons between the pre- and postsurgery scores (matched within subjects) were further made to determine on which postsurgery day(s) the score was different from the one before the surgery. The significance level for $t$ statistics was adjusted according to the number of comparisons; the equations, $\alpha_{M}=1-\left(1-\alpha_{N}\right)^{c}$ and $\alpha_{N}=\alpha / C$ [where $\alpha_{M}$ and $\alpha_{N}$ were the adjusted and referred significance levels, respectively, and $\alpha$ and $C$ were the originally chosen significance level (i.e., 0.05) and the number of comparisons, respectively], were used for this adjustment. The $\alpha_{M}$ and $\alpha_{N}$ were 0.0491 and 0.0125 , respectively.
A
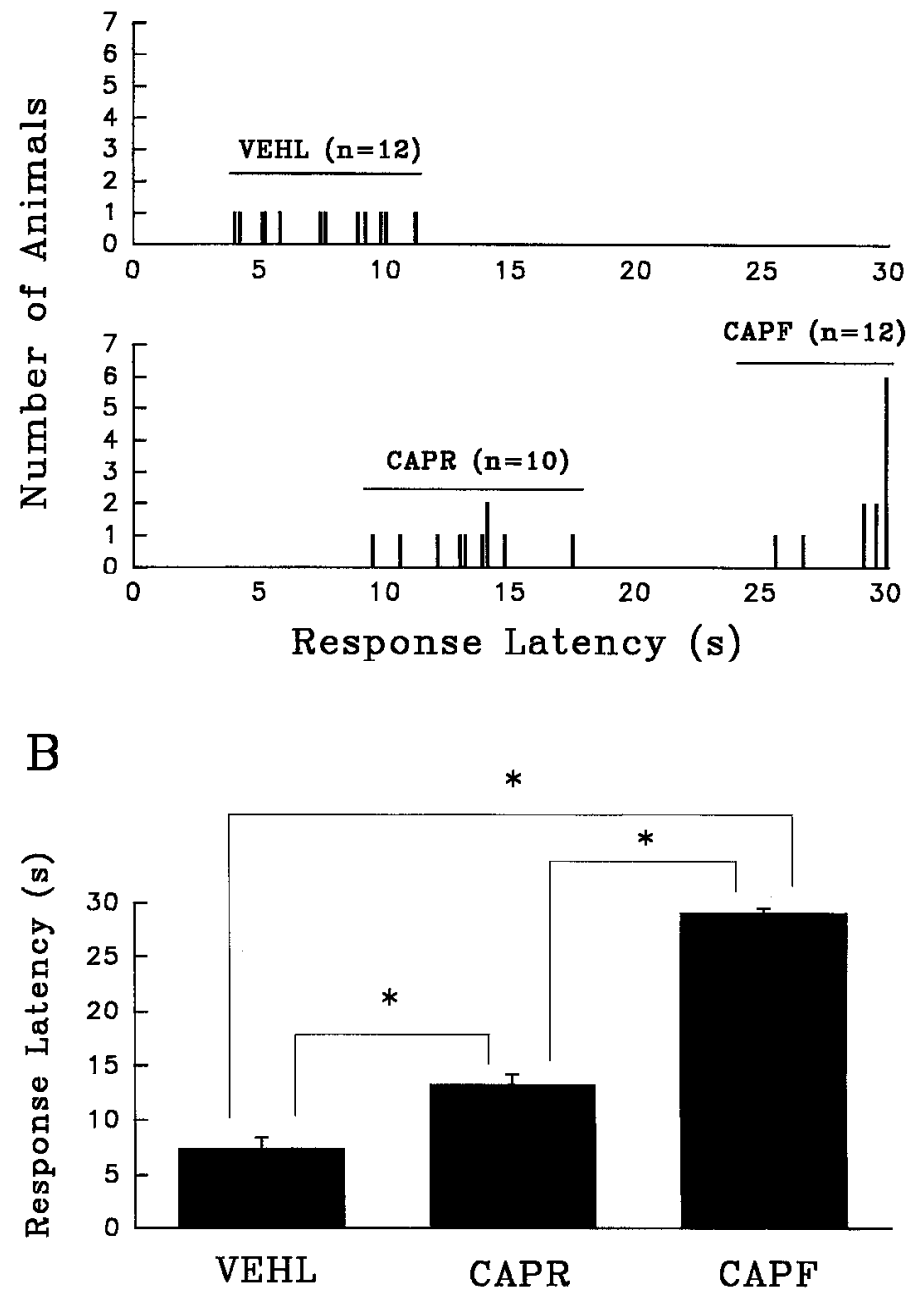

Figure 2. Capsaicin-induced increase in the latency of the tail-withdrawal response to hot $\left(48^{\circ} \mathrm{C}\right)$ water stimulation. $A$, Frequency histograms showing the number of animals with a given tail response latency. $B$, mean $( \pm \mathrm{SEM})$ tail response latency for each animal group. ${ }^{*}, p$ $<0.0001$ (Student $t$ test). VEHL, Vehicle-treated group, CAPR, capsaicin-treated group with a relatively short $(9.5-17.5 \mathrm{sec})$ tail-withdrawal latency; $C A P F$, capsaicin-treated group with a relatively long (25.6-30 $\mathrm{sec})$ tail-withdrawal latency. These abbreviations also apply to the subsequent figures.

The $p$ values in the captions of Figures 3-5 are the ones obtained after considering the adjustment.

Mann-Whitney $U$ test was used to examine whether significant differences were present between animal groups in the test scores obtained on a given experimental day. $p<0.05$ was considered significant.

\section{Results}

\section{Verification of capsaicin effects}

When checked in adulthood, the neonatally capsaicin-injected rats, unlike the vehicle-treated ones, exhibited numerous wounds and scars all over their bodies and faces. This suggests that degeneration of $\mathrm{C}$ - and/or $\mathrm{A} \delta$-fibers did occur after the capsaicin treatment, resulting in impairment of nociception.

The latency of the tail-withdrawal response to the hot $\left(48^{\circ} \mathrm{C}\right)$ water stimulation was significantly longer in capsaicin-injected rats than in vehicle-treated ones (Fig. 2), further suggesting that thermal nociceptive threshold increased as a result of $\mathrm{C}$ - and/or 


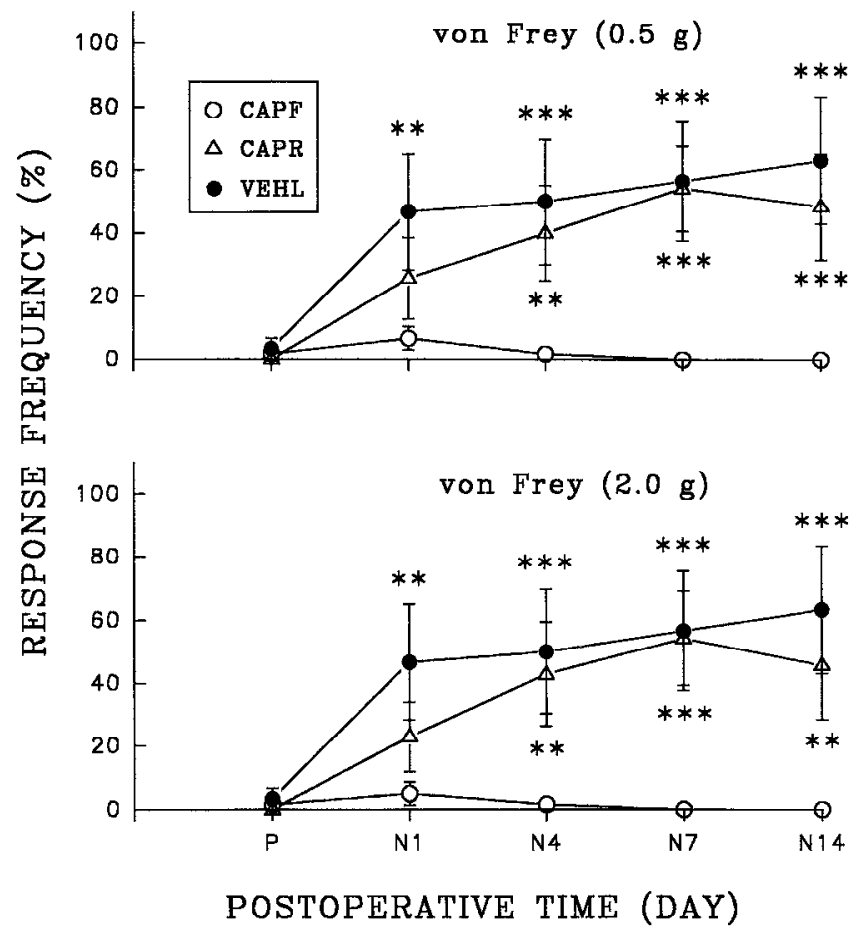

Figure 3. Lack in the CAPF group of the development of mechanical allodynia following the "neuropathic" surgery. Mean ( \pm SEM) frequencies of the abnormal tail responses evoked by $0.5 \mathrm{gm}$ (upper panel) and $2.0 \mathrm{gm}$ (lower panel) von Frey hairs before and after the "neuropathic" surgery are plotted. $P$, Day before the surgery; $N 1, N 4, N 7$, and N14, day $1,4,7$, and 14 after the surgery. These abbreviations also apply to the subsequent figures. The results of the pairwise post hoc comparisons between pre- and postsurgery scores are expressed with the use of asterisks; **, $p<0.01 ; * * *, p<0.001$.

A $\delta$-fiber degeneration following the capsaicin treatment. The tail-withdrawal response latency in capsaicin-injected rats exhibited a bimodal distribution (Fig. $2 A$ ). Since this bimodality was suggestive of variability among animals in the amount of destruction of unmyelinated fibers by capsaicin (Nagy and Van der Kooy, 1983), we divided the capsaicin-treated animals into two groups, namely, CAPR and CAPF (Fig. 2A). The latencies of the CAPR and CAPF groups ranged 9.5-17.5 sec and 25.6-30 sec, respectively. Student $t$ test indicated that the mean latency of the CAPR group ( $12.7 \pm 0.9 \mathrm{sec}, n=10)$ was significantly shorter than that of the CAPF group $(29.1 \pm 0.4 \mathrm{sec}, n=12$, $p<0.0001)$, but was longer than that of the vehicle-treated group (VEHL, $7.4 \pm 1.0 \mathrm{sec}, n=12, p<0.0001$ ) (Fig. $2 B$ ).

\section{Neuropathic pain behaviors}

Mechanical allodynia. Prior to the "neuropathic" surgery, the frequency of the abnormal tail response to von Frey hair stimulation was near $0 \%$, regardless of the capsaicin treatment status (Fig. 3). When checked after the surgery, the frequency increased dramatically in the VEHL and CAPR groups but not in CAPF group (Fig. 3), suggesting that the surgery led to the development of mechanical allodynia only in the VEHL and CAPR groups; Friedman's rank test revealed that a significant difference was present among the behavioral test scores obtained on pre- and postsurgery days in the VEHL $(p<0.0001$ for both $0.5 \mathrm{gm}$ and $2.0 \mathrm{gm}$ von Frey hair tests) and CAPR $(p<0.0001$ for both $0.5 \mathrm{gm}$ and $2.0 \mathrm{gm}$ von Frey hair tests) groups, whereas the test indicated no significant difference among the scores in
Table 1. Between-group comparisons by Mann-Whitney $U$ test in the behavioral test scores

Pre- Post- Post- Post- Postsurgery surgery surgery surgery surgery (day 1) (day 1) (day 4) (day 7) (day 14)

a. $0.5 \mathrm{gm}$ von Frey hair test

VEHL vS CAPF NS NS ** * * *

VEHL vS CAPR NS NS NS NS NS

CAPF vs CAPR NS NS * * *** *

b. $2.0 \mathrm{gm}$ von Frey hair test

VEHL vS CAPF NS NS ** * * * **

VEHL VS CAPK NS NS NS NS NS

CAPF vs CAPR NS NS NS *** *

c. Cold water test

VEHL vs CAPF NS NS NS NS *

VEHL vS CAPR NS NS NS NS *

CAPF vs CAPR NS NS NS NS NS

d. Warm water test

VEHL vs CAPF

VEHL vs CAPR

CAPF vs CAPR

$\begin{array}{lllll}\text { NS } & * * & \text { NS } & * & * * \\ \text { NS } & * & \text { NS } & \text { NS } & * * \\ \text { NS } & \text { NS } & \text { NS } & \text { NS } & \text { NS }\end{array}$

$\overline{\text { VEHL, vehicle-treated group; CAPR, capsaicin-treated group with a relatively }}$ short $(9.5-17.5 \mathrm{sec})$ latency in the tail-withdrawal response to hot $\left(48^{\circ} \mathrm{C}\right)$ water stimulation; CAPF, capsaicin-treated group with a relatively long $(25.6-30 \mathrm{sec})$ latency. $*, p<0.05 ; * *, p<0.01 ; * * *, p<0.001$; NS, not significantly different.

the CAPF group $(p>0.15$ for $0.5 \mathrm{gm}$ and $p>0.49$ for $2.0 \mathrm{gm}$ von Frey hair tests). Pairwise post hoc comparisons further revealed that, regardless of the von Frey hair force, the scores on postsurgery days $1,4,7$, and 14 in the VEHL group and the scores on postsurgery days 4,7 , and 14 in the CAPR group were significantly higher than the corresponding presurgical scores (Fig. 3). In addition, Mann-Whitney $U$ tests indicated that, in the case of the $0.5 \mathrm{gm}$ von Frey hair test (Table 1a), the scores on postsurgery days 4,7 , and 14 of the VEHL and CAPR groups were significantly higher than the scores of the CAPF group on the corresponding days and, in the case of the $2.0 \mathrm{gm}$ von Frey hair test (Table 1b), the scores on postsurgery days 4, 7 , and 14 of the VEHL group and the scores on postsurgery days 7 and 14 of the CAPR group were significantly higher than the scores of the CAPF group on the corresponding days. On the other hand, Mann-Whitney $U$ tests revealed no significant difference between the scores of the VEHL and CAPR groups on any given experimental day (Table 1a,b).

Thermal allodynia. Bcforc the "ncuropathic" surgery, most rats in both vehicle- and capsaicin-treated groups did not show the abnormal tail responses to the cold $\left(4^{\circ} \mathrm{C}\right)$ or warm $\left(40^{\circ} \mathrm{C}\right)$ water stimuli; only a few rats showed the abnormal responses within $30 \mathrm{sec}$, the cut-off time employed in this study. After the "neuropathic" surgery, the rats in the VEHL group, but not in CAPR and CAPF groups, exhibited the tail responses of significantly decreased latencies (Fig. 4, cold; Fig. 5, warm), suggesting that the surgery resulted in the development of thermal allodynia only in the VEHL group; Friedman's rank test revealed that a significant difference was present among the tail response latencies obtained on pre- and postsurgery days in the VEHL group ( $p<0.01$ for both cold and warm water tests), but not in the CAPR and CAPF groups $(p>0.4)$. Pairwise post hoc comparisons further revealed that, in the VEHL group, the tail response latency on postsurgery day 14 in the case of the cold 


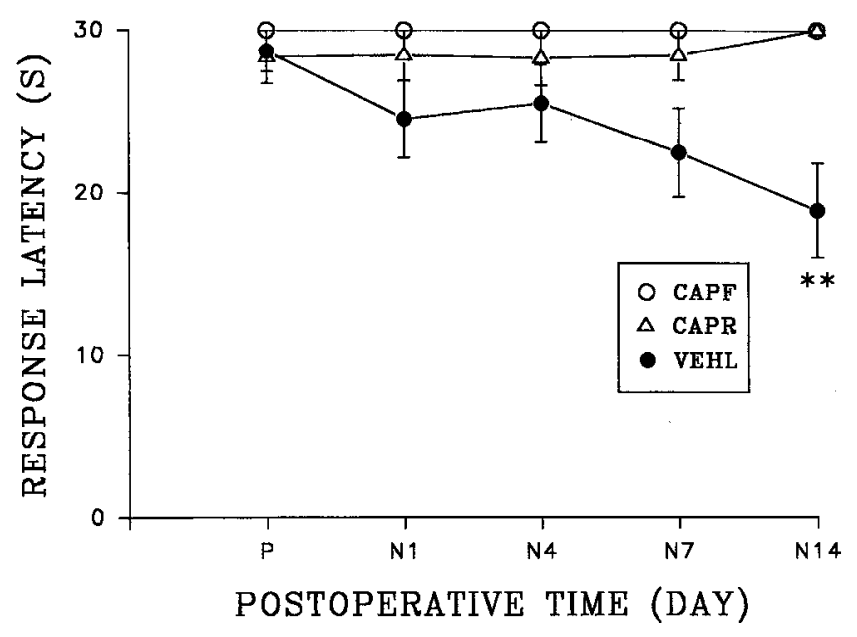

Figure 4. Lack in the CAPR and CAPF groups of the development of cold allodynia following the "neuropathic" surgery. Mean ( \pm SEM) latencies of the tail responses to cold $\left(4^{\circ} \mathrm{C}\right)$ water stimulation before and after the "neuropathic" surgery are plotted. ${ }^{* *}, p<0.01$.

water test (Fig. 4) and the latencies on postsurgery days 1, 7, and 14 in the case of the warm water test (Fig. 5) were significantly shorter than the corresponding presurgical scores. In addition, Mann-Whitney $U$ tests indicated that, in the case of the cold water test (Table 1c), the tail response latency of the VEHL group on postsurgery day 14 was significantly shorter than the latencies of the CAPR and CAPF groups on the same day and, in the case of the warm water test (Table 1d), the latencies of the VEHL group on postsurgery days 1,7 , and 14 were significantly shorter than those of the CAPR and/or CAPF groups on the corresponding days. Mann-Whitney $U$ tests, on the other hand, indicated that the latencies of the CAPR and CAPF groups were not significantly different on any given experimental day (Table 1c,d).

\section{Discussion}

The results of the present study clearly demonstrate that both thermal and mechanical allodynia, produced by transecting some but not all of the nerves innervating the tail, were blocked by a neonatal capsaicin injection, in so far as this treatment resulted in a large increase in the latency of the tail-withdrawal response to hot $\left(48^{\circ} \mathrm{C}\right)$ water stimulation (CAPF group). These results strongly suggest that capsaicin-sensitive $A \delta$ - and/or C-fibers are critical in the induction or expression of the causalgic symptoms.

Our data are in line with the findings of Meller, Gehhart, and Maves (1992) and Shir and Seltzer (1990) that thermal hyperalgesia, produced by a partial sciatic nerve injury (Seltzer et al., 1987, 1990; Bennett and Xie, 1988), was prevented by a similar capsaicin treatment. However, the data are inconsistent with the results of Shir and Seltzer (1990) that mechanical allodynia survived capsaicin treatment, which led these investigators to conclude that mechanical allodynia develops even in the absence of most C-fibers.

The difference in animal model (i.e., hind paw vs tail model) might be a possible explanation for why mechanical allodynia was present in Shir and Seltzer's, but not our, animals. However, the observations that the signs of neuropathic pain in both models were similarly blocked by sympathectomy (Seltzer and Shir, 1991; unpublished observation for our model) do not support the idea.

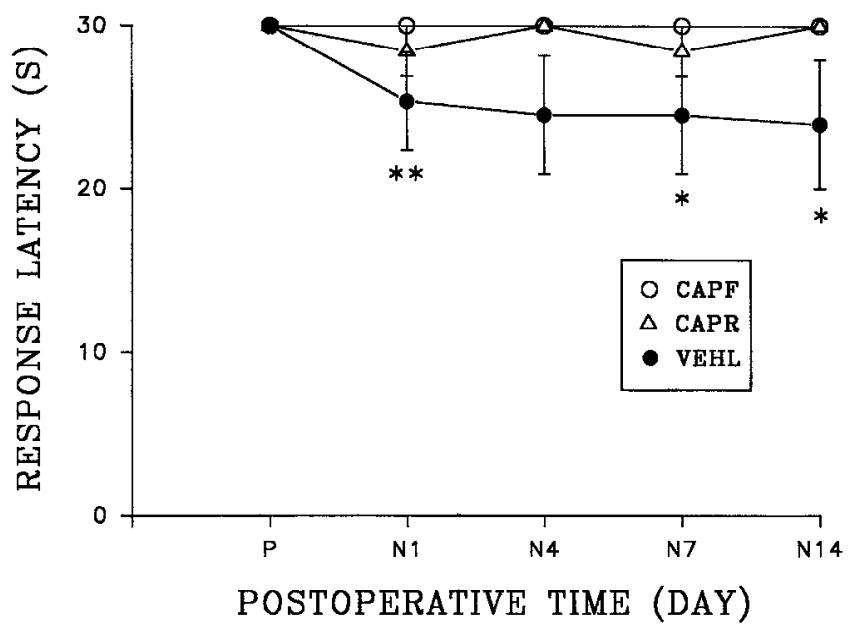

Figure 5. Lack in the CAPR and CAPF groups of the development of warm allodynia following the "neuropathic" surgery. Mean ( \pm SEM) latencies of the tail responses to warm $\left(40^{\circ} \mathrm{C}\right)$ water stimulation before and after the "neuropathic" surgery are plotted. *, $p<0.05 ; * *, p<$ 0.01 .

The discrepancy between our and Shir and Seltzer's results might rather be due to the difference in rat strain (SpragueDawley vs Sabra). Alternatively, it might be due to the difference in the extent of destruction by capsaicin of small diameter fibers. Two different lines of evidence support this view. First, it has been reported that the amount of destruction of unmyelinated sensory fibers by capsaicin is variable between animals (Nagy and Van der Kooy, 1983). Second, in the CAPF group which had no mechanical allodynia, the increase (about 300\%) in the latency of the tail-withdrawal response to hot water stimulation, an indirect measure employed in this study to estimate the degree of destruction of small diameter fibers, was much greater than that (about 70\%) in the CAPR group which showed mechanical allodynia. In this context, it is interesting that in the Shir and Seltzer's study (1990) the capsaicin-induced increase in the thermal nociceptive threshold, another indirect measure for the efficacy of capsaicin in destroying small diameter fibers, was only about $50 \%$.

Based on the present results alone, we cannot draw any firm conclusions for the mechanism(s) underlying the inhibitory effects of capsaicin on the mechanical and thermal allodynia following the nerve injury. One possible mechanism, however, is that the destruction by capsaicin of $\mathrm{A} \delta$ - and $\mathrm{C}$-fibers prevented the activities induced in these fibers by the nerve injury from producing "central sensitization," central changes thought to be required for the emergence of neuropathic pain symptoms following peripheral nerve injury (Roberts, 1986; Coderre et al., 1993). Another possible mechanism is that the capsaicin destruction of $\mathrm{A} \delta$ - and $\mathrm{C}$-fibers disallowed small diameter fiber-mediated sensory signal transmission from the periphery to the central nervous system which is essential in triggering neuropathic pain. This second mechanism would be applicable to the case of the thermal allodynia prevention by capsaicin. Because, removal of small diameter fibers by capsaicin in normal animals is thought to compromise thermal nociception (Fig. 2; Holtzer et al., 1979; Jansco and Jansco-Gabor, 1980; Nagy et al., 1980; Gamse, 1982; Nagy and Van der Kooy, 1983; Doucette et al., 1987; but see Hayes et al., $1981 \mathrm{a}, \mathrm{b}$; Cervero and McRitchie, 1981, 1983; Buck et al., 1982; Hara et al., 1984; Meller ct al., 1992). But, this mechanism would not be applicable to the case 
of the prevention of mechanical allodynia by capsaicin. Because, the von Frey hairs that we used (4.9 and $19.6 \mathrm{mN}$ ) would normally not activate nociceptors with $\mathrm{A} \delta$ - and C-fibers (Leem et al., 1993), low threshold mechanoreceptors with C-fibers are rare in normal rat cutaneous nerves (Fleisher et al., 1983), and therefore, most mechanical sensory information would be carried via capsaicin-insensitive myelinated A-fibers. Thus, as an explanation for the inhibitory effect of capsaicin on the mechanical allodynia, the first mechanism (i.e., the prevention of the central sensitization by capsaicin-induced destruction of $\mathrm{A} \delta$ - and $\mathrm{C}$-fibers), not the second, appears to be appropriate.

In a recent report on the effects of capsaicin on the causalgic symptoms, Shir and Seltzer (1990) suggested that, in the absence of most C-fibers, injury discharges of $\mathrm{A}$-fibers with large diameters are sufficient to produce the central changes required for touch evoked (i.e., mechanical) allodynia. This conclusion contradicts our deduction that the preclusion of the central sensitization by the capsaicin-induced destruction of small diameter fibers was the reason for the lack of mechanical allodynia (see above). To date, no concrete evidence is available for the idea that A-fibers with large diameters could sensitize dorsal horn cells, although the demonstration of the $A \beta$-fiber sprouting into laminae I and II in the spinal dorsal horn following peripheral nerve injury (Woolf et al., 1992; Shortland and Woolf, 1993; Koerber et al., 1994) points to the possibility. On the other hand, there are numerous reports that brief activities of $\mathrm{C}$-nociceptors resulted in prolonged changes in the excitability of certain dorsal horn cells (e.g., wide dynamic range cells) (Price and Browe, 1975; Price et al., 1978; Chung et al., 1979; Kenshalo et al., 1979, 1982; Schouenborg and Sjolund, 1983), rendering the gains of these neurons to both A- and C-fiber inputs enhanced (Kenshalo et al., 1982; Schouenborg and Sjolund, 1983). Conceivably, the mechanical allodynia in capsaicin treated animals in Shir and Seltzer's study (1990) was the result of a central sensitization produced hy small diameter fihers that were not destroyed by capsaicin, not by capsaicin-insensitive A-fibers with large diameters. Similarly, it is possible that the mechanical allodynia in the CAPR group in our study resulted from an incomplete destruction of small diameter fibers by capsaicin which permitted a central sensitization sufficient for the mechanical allodynia.

In summary, the present results strongly suggest that capsaicin-sensitive small diameter fibers such as $\mathrm{C}$ - or $\mathrm{A} \delta$-fibers play a critical role in the development of both the mechanical and thermal allodynia. The results further suggest that the central change induced by injury discharges of small diameter fibers is a prerequisite for the mechanical allodynia.

\section{References}

Bennett GJ, Xie Y-K (1988) A peripheral mononeuropathy in rat that produces disorders of pain sensation like those seen in man. Pain 33: $87-107$.

Blumberg H, Janig W (1983) Changes of reflexes in vasoconstrictor neurons supplying the cat hind limb following chronic nerve lesions: a model for studying mechanisms of reflex sympathetic dystrophy. J Auton Nerv Sys 7:399-411.

Buck SH, Miller MS, Burks TF (1982) Depletion of primary afferent substance $\mathbf{P}$ by capsaicin and dihydrocapsaicin without altered thermal sensitivity in rats. Brain Res 233:216-220.

Cervero F, McRitchie HA (1981) Neonatal capsaicin and thermal nociception: a paradox. Brain Res 215:414-418.

Cervero F, McRitchie HA (1983) Effects of neonatal administration of capsaicin on several nociceptive systems. Adv Pain Res Ther 5:8794.

Chung JM, Kenshalo DR, Gerhart KD, Willis WD (1979) Excitation of primate spinothalamic ncurons by cutancous C-fibers volleys. J Neurophysiol 42:1354-1369.

Coderre TJ, Katz J, Vacarrino AL, Melzack R (1993) Contribution of central neuroplasticity to pathological pain: review of clinical and experimental evidence. Pain 52:259-285.

Devor M (1983) Nerve pathophysiology and mechanisms of pain in causalgia. J Auton Nerv Sys 7:371-384.

Doucette E, Theriault E, Diamond J (1987) Regionally selective elimination of cutaneous thermal nociception in rats by neonatal capsaicin. J Comp Neurol 261:583-591.

Fleisher E, Handwerker HO, Joukhadar S (1983) Unmyelinated nociceptive units in two areas of the rat. Brain Res 267:81-92.

Gamse R (1982) Capsaicin and nociception in the rat and mouse. Possible role of substance P. Naunyn Schmiedebergs Arch Pharmacol 320:205-216.

Hara A, Sakurada T, Sakurada S, Matsumura H, Kisara K (1984) Antinociceptive effects of neonatal capsaicin in rats with adjuvant arthritis. Naunyn Schmiedebergs Arch Pharmacol 326:248-253.

Hayes AG, Scadding JW, Skingle M, Tyers MB (1981a) Effects of neonatal administration of capsaicin on nociceptive thresholds in the mouse and rats. J Pharm Pharmacol 33:183-185.

Hayes AG, Skingle M, Tyers MB (1981b) Effects of single doses of capsaicin on nociceptive thresholds in the rodent. Neuropharmacology 20:505-511.

Holtzer P, Jurna I, Gamse R, Lembeck F (1979) Nociceptive threshold after neonatal capsaicin treatment. Eur J Pharmacol 58:51 1-514.

Jansco G, Jansco-Gabor A (1980) Effect of capsaicin on morphine analgesia-possible involvement of hypothalamic structures. Naunyn-Schmeideberg's Arch Pharmacol 311:285-288.

Kenshalo DR, Leonard RB, Chung JM, Willis WD (1979) Responses of primate spinothalamic neurons to graded and to repeated noxious heat stimuli. J Neurophysiol 42:1370-1389.

Kenshalo DR, Leonard RB, Chung JM, Willis WD (1982) Facilitation of the responses of primate spinothalamic cells to cold and mechanical stimuli by noxious heating of the skin. Pain 12:141-152.

Kim SH, Chung JM (1991) Sympathectomy alleviates mechanical allodynia in an experimental animal model for neuropathy in the rat. Neurosci Lett 134:131-134.

Kim SH, Chung JM (1992) An experimental model for peripheral neuropathy produced by segmental spinal nerve ligation in the rat. Pain 50:355-363.

Koerber HR, Mirnics K, Brown PB, Mendell LM (1994) Central sprouting and functional plasticity of regenerated primary afferents. J Neurosci 14:3655-3671.

Lawson SN, Nickels SM (1980) The use of morphometric techniques to analyze the effect of neonatal capsaicin treatment on rat dorsal root ganglia and dorsal roots. J Physiol (Lond) 303:12P.

Leem JW, Willis WD, Chung JM (1993) Cutaneous sensory receptors in the rat foot. J Neurophysiol 69:1684-1699.

Meller ST, Gebhart GF, Maves TJ (1992) Neonatal capsaicin treatment prevents the development of the thermal hyperalgesia produced in a model of neuropathic pain in the rat. Pain 51:317-321.

Mitchell SW (1872) Injuries of nerves and their consequences. Philadelphia: Lippincott.

Na HS, Han JS, Ko KH, Hong SK (1994) A behavioral peripheral neuropathy produced in rat's tail by inferior caudal trunk injury. Neurosci Lett 177:50-52.

Nagy JI, Van Der Kooy (1983) Effects of neonatal capsaicin treatment on nociceptive thresholds in the rat. J Neurosci 3:1145-1150.

Nagy JI, Vincent SR, Staines WA, Fibiger HC, Reisine TD, Yamamura HI (1980) Neurotoxic action of capsaicin on spinal substance P neurons. Brain Res 186:435-444.

Nagy JI, Emson P, Iversen LL (1981) A re-evaluation of the neurochemical and antinociceptive effects of intrathecal capsaicin in the rat. Brain Res 211:497-502.

Price DD, Browe AC (1975) Spinal cord coding of graded non-noxious and noxious temperature increases. Exp Neurol 48:201-221.

Price DD, Hayes RL, Ruda M, Dubner R (1978) Spatial and temporal transformations input to spinothalamic tract neurons and their relation to somatic sensation. J Neurophysiol 41:933-946.

Richards RS (1967) Causalgia. Arch Neurol 16:339-350.

Roberts WJ (1986) A hypothesis on the physiological basis for causalgia and related pains. Pain 24:297-311.

Schouenborg J, Sjolund BH (1983) Activity evoked by A- and C- 
afferent fibers in the rat dorsal horn neurons and its relation to a flexion reflex. J Neurophysiol 50:1108-1120.

Seltzer Z, Shir Y (1991) Sympathetically-maintained causalgiform disorders in a model for neuropathic pain: a review. $J$ Basic Clin Physiol Pharmacol 2:17-61.

Seltzer Z, Dubner R, Shir Y (1987) Bilateral hyperesthesia, bilateral hyperalgesia and unilateral hyperpathia in rats following partial nerve injury. Rev Clin Bas Pharmacol 6:375.

Seltzer Z, Dubner R, Shir Y (1990) A novel behavioral model of neuropathic pain disorders produced in rats by partial sciatic nerve injury. Pain 43:205-218.
Shir Y, Seltzer Z (1990) A-Fibers mediate mechanical hyperesthesia and allodynia and $\mathrm{C}$-fibers mediate thermal hyperalgesia in a new model of causalgiform pain disorders in rats. Neurosci Lett 115:6267.

Shortland P, Woolf CJ (1993) Chronic peripheral nerve section results in a rearrangement of the central axonal arborizations of axotomized A beta primary afferent neurons in the rat spinal cord. $J$ Comp Neurol 330:65-82.

Tahmoush AJ (1981) Causalgia: redefinition as a clinical pain syndrome. Pain 10:187-197.

Woolf CJ, Shortland P, Coggeshall RE (1992) Peripheral nerve injury triggers central sprouting of myelinated afferents. Nature 355:75-78. 